\title{
Development of models to assess the environmental impacts arising from chronic atmospheric and sub-surface sources of tritium
}

\author{
Y. Belot, P. Davis ${ }^{1}$, A. Golubev ${ }^{2}$, G. Guinois ${ }^{3}$, M. Taeschner ${ }^{4}$ and B. Watkins ${ }^{5}$ \\ Independent Consultant, France \\ ${ }^{1}$ AECL, Canada \\ ${ }^{2}$ RFNC-VNIIEF, Russia \\ ${ }^{3}$ CEA/DASE, France \\ ${ }^{4}$ ZSR, Germany \\ ${ }^{5}$ QuantiSci Ltd. U.K.
}

\begin{abstract}
Models that describe tritium dispersion in the atmosphere and its subsequent transfer to soil and vegetation were compared through use of well-defined scenarios, and validated by comparing predictions with observed long-term data. Predicted concentrations agreed with observations to within a factor of 2-4 for all compartments (air, soil, vegetation). A comparison of sub-surface models for predicting tritium migration into or out of aquifers, was also carried out, and it was shown that in each of the cases different models agree within a factor of 2 for most endpoints. Validation of the subsurface models against observations is still to be done.
\end{abstract}

In the framework of the IAEA-coordinated BIOMASS programme, models to assess the impact of chronic releases of tritium were intercompared and tested. Eleven Institutions from different countries participated to the work (a full list of participants can be seen at the end of the text). The present paper summarises the modelling methods and the main results obtained. A complete report of the work is given in [1].

\section{DISPERSION IN THE ATMOSPHERE}

\subsection{HTO releases}

Tritium concentrations in air humidity were determined either by traditional models of atmospheric dispersion, or by specially developed models that consider both primary emission from main sources and secondary emission from the contaminated surface. All these models generally use the statistical sector-averaged Gaussian formula, based on wind direction sectors centred at the source.

Fig. 1 (left) shows the concentrations in air humidity that have been obtained for an hypothetical scenario that assumes an annual release of $1 \mathrm{~g}$ of tritium as HTO, through a 60 -m high stack, using statistics of meteorological conditions provided in the scenario description. All but one of the dispersion curves obtained by the eleven participants were within a factor of about 1.7. To ensure legibility of the graph, only the extreme curves are represented. The consideration of re-emission from ground-level sources does not appreciably change the results (less than 10\%), at least for the conditions fixed in the scenario. The rest of the spread is due to variations among models in the values of wind speed and vertical dispersion parameter that are used in the calculation.

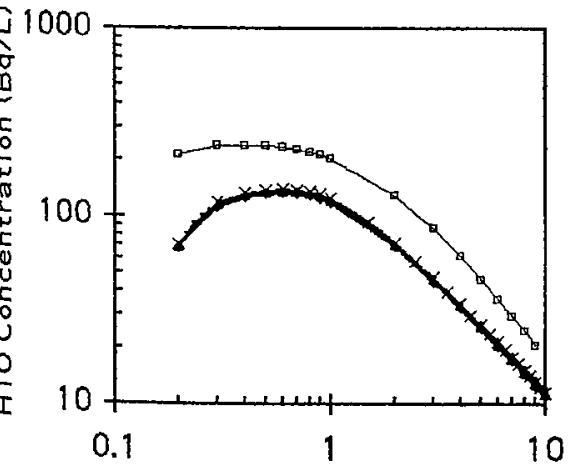

Distance from Source $(\mathrm{km})$

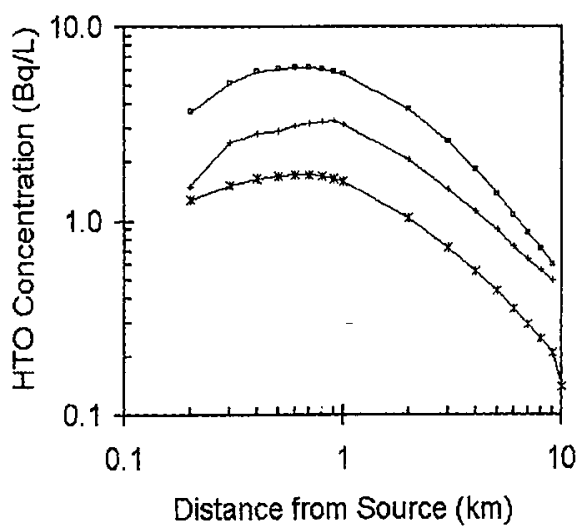

Figure 1: Predicted concentrations of HTO in air humidity for a release of HTO (left) and for a release of HT (right ). In both cases, the tritium release rate is $1 \mathrm{~g} \mathrm{a}^{-1}$. Most of the results are between the extreme upper and lower curves 
To validate the models, blind test exercises were performed in which predictions from the different models were compared with observations made on Canadian, Russian and French sites. In most cases predictions agreed with the observed air concentrations within a factor of three for the Canadian and French sites, and within a factor of four for the Russian site. The results are considered acceptable for the scenarios based on Canadian and French data. The more divergent results for the Russian site were due to the unavailability of complete input data.

The Valduc site in France was particularly interesting because it is situated in a rolling terrain with shallow valleys and moderate slopes, but where the differences in elevation can reach $150 \mathrm{~m}$ or so over several $\mathrm{km}$. Some of the modellers tried to take account of the topography. This was achieved by assuming that the centrelines of the plumes do not follow the profile of the terrain at a constant height above ground level, but tend to remain more or less at a constant elevation above sea level, depending on the atmospheric stability. The results obtained with topographic correction were not significantly better than those obtained without correction.

\subsection{HT releases}

HT releases were only considered in the first model-model intercomparison scenario. Concentrations of HTO in air moisture following an HT release are entirely controlled by oxidation of HT at the soil surface, subsequent re-emission of HTO and dispersion in the atmosphere. In most models, these processes were simulated by estimating the average concentration of HTO in the near-surface soil water and then summing the different contributions from the re-emission process to the concentrations in the atmosphere. An alternative approach was to use the empirical ratios obtained from field experiments held at Chalk River Laboratories (CRL) in Canada [2], assuming that the re-emission is only moderately sensitive to the local soil and weather conditions.

Most of the model predictions were spread over a factor of five and were situated between the extreme curves represented on the graph of Fig.1 (right). The intermediate curve was derived from the CRL empirical ratios. This is one area where modelling efforts should be continued, in parallel to the collection of new experimental data. Until this is forthcoming, it is recommended that the empirical ratios derived from the $1994 \mathrm{HT}$ chronic release experiment conducted at CRL are used, namely that HTO in air $\left.(\mathrm{Bq} \mathrm{m})^{-3}\right)$ due to an $\mathrm{HT}$ release is 0.04 times the predicted $\mathrm{HT}$ concentration in air $\left.(\mathrm{Bq} \mathrm{m})^{-3}\right)$.

\section{TRANSFER TO SOIL AND VEGETATION}

\subsection{Deposition to soil surface}

In the case of an HTO or HT release, the estimations of average tritium concentration in the near-surface soil water were obtained in different ways. Certain modellers used an activity balance equation for the flux of tritium into and out of the soil, taking into account wet and dry deposition, re-emission and infiltration. Some assessed the contribution of tritium wet and dry deposition using a semi-empirical approach, assuming that the concentration in soil water equals the wet flux plus an arbitrary fraction of the dry flux, the sum of both fluxes being divided by the precipitation rate. Others considered that the contribution of the dry flux was negligible, and that the concentration in soil water was simply equal to the concentration in rainwater.

Different predictions arising from the approaches outlined above were compared to observations made at CRL, where HTO concentrations had been measured twice daily in air, soil and vegetation at different locations during a period of two months. Predictions of the activity balance models, expressed as soil to air ratios, were a factor $2-3$ above the observations, as they gave a ratio of 0.6 to 0.8 instead of 0.3 on average. These models attribute about $75 \%$ of the soil tritium inventory to dry deposition, which appears to be too high. They underestimate the amount of tritium re-emitted from the soil to the atmosphere and so overestimate the soil concentration. In addition, the exchange velocities used in these models, which had been determined in earlier experiments, are too high in certain field conditions. Moreover, it is not certain that the assumption of a steady-state regime is valid in a situation where the air concentrations and the deposition fluxes over the sampling sites fluctuate continually in response to changing atmospheric conditions and release rates. The modelling approach that sets the soil concentration equal to the rain concentration underpredicts soil concentrations at all sampling points, which suggests that dry deposition must be considered when estimating soil concentrations. A semiempirical model performed well for conditions at this site, with predictions lying within about $50 \%$ of the observations. This model estimates that dry and wet deposition contribute about equally to the soil tritium inventory.

It was decided to commission a field survey with the specific objective of collecting data on the relative contributions of wet and dry deposition of HTO to soil water concentrations. The work was 
carried out at a site near Bruyeres-le-Chatel, France. Concentrations of HTO were measured monthly in air moisture, rain and soil moisture at two monitoring stations that received either mainly dry or mainly wet deposition. The results of the 20-month sampling programme showed that dry deposition does contribute to soil moisture concentrations. Moreover, the ratio of soil/air concentrations showed a spatial variability that depended on the joint frequency of precipitation occurrence and wind direction. The ratio was 0.2 at a monitoring station situated at the East of the source (Fig.2) and about 0.1 at the West, due to a significant difference in the occurrence of contaminated rainfall at the two stations.

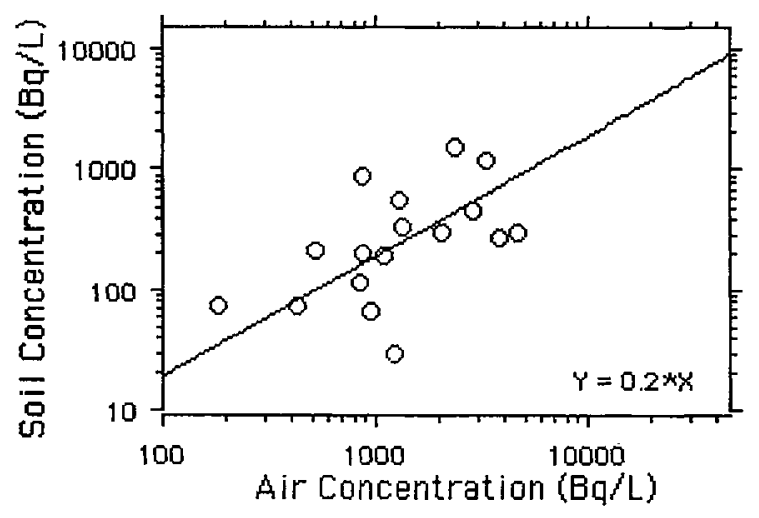

Figure 2: Observed values of end-of-month soil concentrations $(\mathrm{Bq} / \mathrm{L}$ of soil water) vs. monthly-averaged air concentrations (Bg/L of air humidity) at a sampling point which is located at the East of a tritium source in Bruyères-le-Châtel France. The slope of the regression line passing through the origin is 0.2 . The coefficient of determination is $R^{2}=0.511$.

The data collected at Chalk River and Bruyeres le Chatel, provides the first step toward the development of better models for predicting HTO soil concentrations as a result of HTO releases. Until more data are available from other sites, it is suggested that the ratio of soil to air concentrations derived from the above field surveys should be used. Since the soil to air ratio is 0.3 at Chalk River and about 0.2 in Bruyeres le Chatel, it is suggested that a value of 0.3 is used. This simple assumption is more reliable than predictions calculated by more sophisticated models that have been proposed to date. For HT releases, it is recommended that predictions of the HTO concentration in soil can be based on a soil to air empirical ratio of $6 \mathrm{~Bq} \mathrm{~L}^{-1}$ per $\mathrm{Bq} \mathrm{m}^{-3}[2]$.

\subsection{Transfer to vegetation}

Most of the participants based their predictions of HTO concentrations in plant tissue water on the assumption that a fraction RH of leaf water derives from atmospheric water and the remainder (1-RH) derives from the near-surface soil water, where RH is the relative humidity of the atmosphere [3]. The concentration of non-exchangeable organically-bound tritium (OBT) in organic plant material, expressed in activity per unit volume of combustion water, is generally assumed to be $0.6-0.8$ times the concentration of tritium in leaf tissue free water (TFW) [4].

The spread of results for predictions of plant TFW tritium and OBT was smaller than that of results for soil moisture concentrations. Predictions and observations were generally in agreement within a factor of two to three for the model-model inter-comparison and the Canadian and French scenarios, and less than a factor of five for the Russian scenario. This is not surprising, as model calculations depend primarily on air, not soil, concentrations. All the participating models for plant leaf uptake are similar. Overall, the models over-estimated the proportion of HTO taken up from the atmosphere probably because all the leaf water is not entirely accessible to exchange, and moreover the tritium in the leaves fluctuates and turns over rapidly. Generally, the TFWT and OBT over-estimates were relatively small because plant water concentrations are forced by specific activity concepts to be lower than concentrations in air humidity.

The observational data for OBT concentrations in tree leaves and tree rings (in the French scenario) emphasised that information is lacking on the behaviour of OBT in trees. The translocation of 
TFWT and OBT to other plant parts such as tubers, root vegetables, shrub and tree fruits was not considered in the present work, but requires further study, because of its importance in the foodchain.

\section{TRANSPORT INTO GROUNDWATERS}

The interest of the public and regulatory authorities extends beyond the above-ground environment to include the sub-surface media. In particular groundwaters are of interest because they may have become contaminated through previous releases either from atmospheric or sub-surface sources. Hence, groundwaters are considered as a potential source of delayed contamination for the above-ground environment and those living in the vicinity. Consequently, there is a growing interest in estimating the concentration of tritium in groundwaters that have been exposed to contamination over a long period.

A model-model intercomparison exercise was based on a scenario in which an unconfined aquifer situated below an emitting stack was characterised by its longitudinal extension ( $\mathrm{L}=10 \mathrm{~km})$, its hydraulic conductivity $\left(\mathrm{K}=10^{-4} \mathrm{~m} \mathrm{~s}^{-1}\right)$ and recharge rate $\left(\mathrm{w}=150 \mathrm{~mm} \mathrm{a}^{-1}\right)$. The emitting stack was situated at a distance of $5 \mathrm{~km}$ from the flow divide in the direction of groundwater flow. The downward flux of tritium through the water table was assumed to be inversely proportional to the distance from the stack, with a value of $15000 \mathrm{~Bq} \mathrm{a}^{-1}$ at $1 \mathrm{~km}$ from the stack. The modellers were asked to predict the vertical profiles of tritium concentration in the aquifer at different locations and 20 years after the beginning of tritium infiltration into the aquifer, for vertical dispersivities of $0.05 \mathrm{~m}$ and $0.5 \mathrm{~m}$.

The problem was first to determine the steady-state horizontal and vertical flow of water and secondly to determine the downward transient transport of tritium in the aquifer, taking into account the convection-dispersion process and the radioactive decay of tritium. Some modellers adapted flexible numerical models of a commercial type that are normally used to solve a wide range of environmental problems, including flow, heat and mass transport in geological media. Other participants developed their own codes based on process models and numerical techniques formerly developed by others. A few modellers attempted to elaborate analytical models, some of which were based on the use of Green's functions. The analytical models were simpler than the complex models to apply but this was at the expense of some simplification of the problem to be assessed.

From flow models, it was deduced that the mean thickness of the aquifer at the vertical of the stack was $59 \mathrm{~m}$, and that the pore water horizontal velocity was $42 \mathrm{~m} \mathrm{y}^{-1}$. The predicted profiles at $1 \mathrm{~km}$ from the vertical of the stack are shown in Fig. 3 for low and high dispersivities (left and right respectively). Eight out of nine of the profiles, lie close together within a factor of two, between the extreme curves shown on each of the graphs. Only one profile, which corresponds to an oversimplified analytical model in each case, is outside the range so delimited and has not been represented on the graphs. For low dispersifity (left graph), the concentration decreases by a factor of one thousand at a depth of about $40 \mathrm{~m}$ below the water table, which corresponds to a low vertical mixing and a stratification of tritium in the upper part of the aquifer. By contrast, for high dispersivity (right graph), the reduction of concentration is only about a factor of ten at the bottom of the aquifer, which denotes a high vertical mixing. It should be noted that a low value of the vertical dispersivity is more probable than a high value [5].
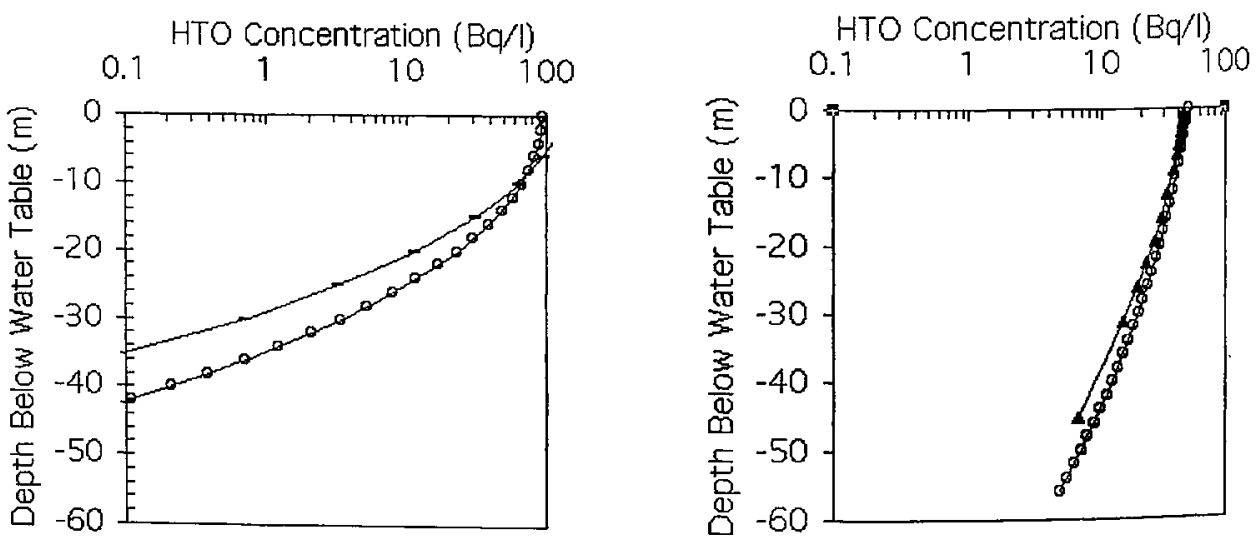

Figure 3: Predicted vertical profiles of HTO in an aquifer at $1 \mathrm{~km}$ from the stack and 20 years after the beginning of tritium infiltration through the water table. The vertical dispersivity of water in the aquifer is $0.05 \mathrm{~m}$ (left) and $0.5 \mathrm{~m}$ (right). Most of the results of the different models lie between the extreme curves shown on the graphs. 
The profiles of Fig. 3 were obtained by imposing a prescribed-flux boundary condition at the water table, which was recognised as more appropriate than the prescribed-concentration condition sometimes used. In the simple yet realistic conditions of the scenario, it was shown that many numerical and analytical models arrived at similar results. But this does not mean that both approaches can be used in any situation, since certain heterogeneities encountered in real situations can only be treated by numerical simulation. The experience gained in the model-model intercomparison showed that the numerical models were rather sensitive to time and space discretisation and that great attention should be paid to this particular point. Moreover, it was shown that the transport of tritium is very sensitive not only to water flow, but also to the value assigned to the dispersivity parameter, which expresses the degree of irregularity of the flow pattern and increases when local velocities vary strongly along the flow path. Experiments using tracers are required to obtain the values of dispersivity to be used in modelling local situations. Default values can be drawn from the literature [5].

\section{TRANSPORT FROM GROUNDWATERS}

Tritium-labelled groundwaters may be a source of contamination for the uncontaminated unsaturated soil layer situated above and for the vegetation rooted in that soil. A model-model inter-comparison exercise was undertaken to address the problem of the upward movement of tritium from a contaminated perched aquifer to the soil surface. Participants were provided with information on water table level, soil characteristics, and hourly meteorological and evapotranspiration data. Modellers were asked to predict the vertical profiles of tritium concentration in the unsaturated part of the soil at specified times after the start of tritium migration, and also the monthly values of the tritiated water vapour flux from the soil surface to the atmosphere. Nine institutions participated in this modelling exercise.

For this specific application, a few modellers adapted multipurpose models that are available commercially. Some developed their own codes based on process models and techniques published by others. For most of these models, the equations of moisture movement and tritium transport in the soil were solved numerically, the linkage between the two equations being performed by calculating the convective water fluxes and the water contents at each time step. One modeller tried to approximate tritium transport by an analytical formula that had been derived from the transport equation assuming constant coefficients and a constant concentration at the water table.

Fig. 4 shows the vertical profile of tritium through the 1m-thick layer of unsaturated soil, for bare and vegetated soils (left and right respectively), in July, seven months after the beginning of tritium upward migration. In the case of bare soil, the concentrations decrease exponentially by 4 orders of magnitude from the water table to the ground surface. In the case of vegetated soil, the concentrations decrease only by $2-3$ orders of magnitude, with some divergence in the results in the near-surface layer. Good agreement among model predictions (a factor of two on average) was obtained at the expense of using numerical models with small time and space increments.

HTO Concentration (Bq/L)

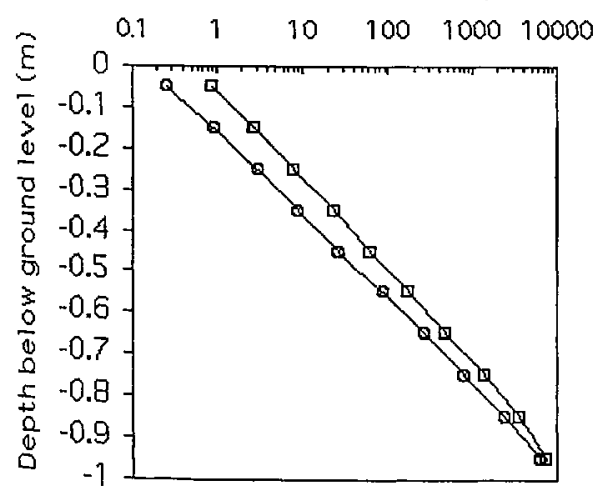

HTO Concentration $(\mathrm{Bq} / \mathrm{L})$

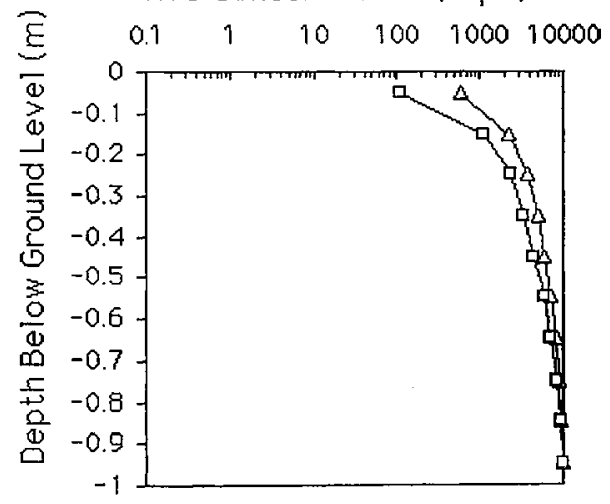

Figure 4: Predicted vertical profiles of HTO in the unsaturated soil above a perched aquifer where the tritium concentration is $10000 \mathrm{~Bq} \mathrm{~L}^{-1}$. The soil is bare (left graph) and vegetated (right graph). The profiles are those obtained at the end of July, seven months after the beginning of tritium upward migration. The tesults from most of the different models lie between the extreme curves shown on the graphs. 
All the modellers agreed and/or showed that the balance of precipitation and evaporation, and the mechanical dispersion process, were the key factors in the transport of tritium in the unsaturated soil layer situated above a shallow water table. The ensemble of results showed that tritium upward transport, although being rather moderate, was significant for the conditions given in the scenario and particularly in the case of a vegetated soil. Any deficit of water in the near-surface soil layer, due to an excess of evapotranspiration over precipitation, induces an upward movement of water through the capillary rise process and simultaneously causes an upward movement of tritium. It is considered that this process is only significant when the water table is very close to the soil surface

\section{CONCLUSIONS}

As concerns the atmospheric pathway, although models are generally satisfactory, there is still some effort required to improve the modelling of tritium deposition and re-emission processes. It is recommended that further work is undertaken to find a more general, theoretically-based model that can account for variations among sites and can quantify properly the concentration of tritium in the nearsurface soil water. It would also be useful to concentrate on the re-emission models, which are indipensable for predicting the air concentrations that are due to ground-level primary or secondary sources. Until new models are developed, the most probable average concentration of tritium in soil water can be taken as equal to 0.3 times the average concentration in air humidity. Experimental investigations in the field should be pursued in parallel to the modelling effort.

The model calculations of the distribution of water concentrations with depth in saturated or unsaturated soil media were made without being able to compare the results with field data. Nevertheless, the use of different models showed the interdependency between the movement of ordinary water and that of tritiated water. Both depend on precipitation, evaporation and the hydraulic properties of the media. The tritium profiles can be determined by the numerical resolution of two coupled equations, by generally using small space intervals. Analytical solutions could also be used, although it should be recognised that the results are only likely to be an approximation and hence are mainly of use for scoping calculations. Most of the numerical codes used for predicting tritium movement in the soil need to be completed by a specific module that calculates more accurately the exchanges of HHO and HTO across the earthatmosphere boundary, taking into account not only the dispersive properties of the near-surface soil, but also those of the atmosphere in contact with the ground surface.

\section{Main Tritium Working Group Participants}

H. Amano (JAERI, Japan); M. Andoh (JAERI, Japan); Y. Belot (Consultant, France); L. Belovodsky, (VNIIEF, Russian Federation); P. Davis (AECL, Canada); O. Edlund (Studsvik, Sweden); D. Galeriu (NIPNE, Romania); A. Golubev (VNIIEF, Russian Federation); G. Guinois (CEA, France); Y. Inoue (IAEA, Austria); D. Lush (BEAK, Canada); S. Mavrin (VNIIEF, Russian Federation); C. Meurville (ANDRA, France); R. Peterson (LLNL, USA); W. Raskob (FZK, Germany); M. Täschner (ZSR, University of Hannover, Germany); B. Watkins (QuantiSci, UK); H. Yamazawa (JAERI, Japan).

\section{Acknowledgments}

The authors wish to thank H.Camus, D.Delmont and G.Brachet, from CEA, France, for their encouragments and support.

\section{References}

[1] Tritium Working Group TECDOC, Modelling the Environmental Transport of Tritium in the Vicinity of Long-term Atmospheric and Sub-Surface Sources, IAEA, Vienna 2001, 356 pp.

[2] Davis P.A. and Bickel G.A. "Environmental HTO/HT Ratios Arising from a Chronic Atmospheric HT Release",Environmental Behavior and Biological Effects of Tritium, Kumatori, Osaka, Japan, 8-9 May 2000, M. Saito, S. Kimura and T. Takahashi Eds (Reasearch Reactor Institute, Kyoto University)

[3] Murphy C.E., Jr, Health Physics 47 (1984) 635-639

[4] Kim M.A. and Baumgärtner F. , Appl. Radiat.. Isotopes 45 (1994) 353-360

[5] Gelhar L.W., Welty C. and Rehfeldt K.R., Water Resour. Res. 28 (1992), 1955-1974. 